\title{
Teoria do Raio Espectral e o Esforço de Vacinação Mínimo para Infecções de Transmissão Direta
}

\author{
C.H. DEZOTTI ${ }^{1}$, Depto de Matemática, CCE, UFRN e Depto de Matemática \\ Aplicada, IMECC, UNICAMP, Campinas, SP, Brasil
}

H.M. YANG², Depto de Matemática Aplicada, IMECC, UNICAMP.

\begin{abstract}
Resumo. Neste trabalho estabelecemos uma caracterização do número de reprodutibilidade basal $R_{0}$ para uma doença infecciosa de transmissão direta como sendo o raio espectral da derivada de Fréchet de um operador integral. Obtemos limites inferior e superior para $R_{0}$ e condições suficientes para a unicidade da solução nãotrivial da força de infecção, que neste caso pode ser atingida como o limite de uma seqüência recursiva. Como aplicação, consideramos uma taxa de contato constante em todas as idades e obtivemos resultados clássicos bem como o esforço mínimo de vacinação necessário para a erradicação da doença.
\end{abstract}

\section{Introdução}

Um parâmetro de interesse em modelos epidemiológicos é o número de reprodutibilidade basal $R_{0}$. Ele representa a capacidade intrínseca que um microorganismo tem de invadir e se estabelecer em uma comunidade. No caso de doenças infecciosas de transmissão direta, onde o agente etiológico é um microparasita, $R_{0}$ pode ser definido como o número total de infecções secundárias que um único indivíduo infeccioso é capaz de produzir em uma população hospedeira totalmente suscetível. Assim, se $R_{0} \leq 1$ a doença se extingue e se $R_{0}>1$ temos a infecção em nível endêmico. A dificuldade na obtenção direta de $R_{0}$ deve-se ao fato dele reunir em si não só as características biológicas da doença e físicas do ambiente, mas também englobar o comportamento social da comunidade onde tenta se estabelecer.

Se a população for assumida homogeneamente misturada, ou seja, os indivíduos não são distingüidos do ponto de vista epidemiológico, segue da Lei de Ação das Massas uma equação que ao mesmo tempo fornece uma maneira de estimarmos o número de reprodutibilidade basal e uma condição para controle epidemiológico da doença (Anderson e May [1]). Esta equação é dada por

$$
1=R_{0} x^{*}
$$

\footnotetext{
${ }^{1}$ dezotti@ime.unicamp.br

2hyunyang@ime.unicamp.br
} 
onde $x^{*}$ representa a fração de indivíduos suscetíveis no equilíbrio (note que $x^{*}$ pode ser efetivamente encontrado, por exemplo, a partir de estudos sorológicos).

No entanto, a asserção de ser a população homogeneamente misturada é muito restritiva e efetivamente não se verifica no caso, por exemplo, de doenças infecciosas de transmissão direta, como rubéola, sarampo ou catapora. Estas doenças infantis têm visivelmente uma idade-dependência na sua taxa de transmissão. Desta maneira, um modelo mais realista para doenças infecciosas de transmissão direta deve conter algum tipo de heterogeneidade. Essa não-homogeneidade foi introduzida na taxa de transmissão de várias maneiras [1], [6], [7], [8] e [12]), e a partir desta asserção vários resultados acerca do número de reprodutibilidade basal $R_{0}$ foram deduzidos.

Greenhalgh [5] e Inaba [8] caracterizaram $R_{0}$ como sendo o raio espectral de um operador integral, respectivamente, para funções separáveis e um subconjunto especial de $\mathcal{L}^{1}[0, L]$, onde $L$ representa a idade máxima de vida. Em nosso trabalho utilizamos um modelo idade-estruturado e caracterizamos $R_{0}$ como sendo o raio espectral da derivada de Fréchet de um operador integral agindo no espaço de Banach de funções contínuas sobre $[0, L]$ e obtemos limites inferior e superior para $R_{0}$. Estabelecemos condições suficientes para a unicidade do estado estacionário não-trivial e uma maneira recursiva para obtenção da força de infecção. Considerando uma taxa de contato constante para todas as idades re-obtemos resultados clássicos e uma forma de calcular o esforço mínimo de vacinação $\nu^{\text {th }}$ necessário para a erradicação da doença.

\section{O modelo}

Para descrever o espalhamento de uma doença infecciosa de transmissão direta em uma população idade-estruturada, consideramos um sistema de equações integrodiferenciais. Assumimos a população fechada e dividida em compartimentos designados por $X(a, t), H(a, t), Y(a, t)$ e $Z(a, t)$, que representam, respectivamente, a densidade de indivíduos suscetíveis, latentes (infectados mas não infecciosos), infecciosos e imunes na idade $a$ no instante $t$. O tamanho total da comunidade é assumido constante de modo que a taxa de mortalidade $\mu$ e a taxa de nascimento $X_{b}$ devem satisfazer a equação

$$
X_{b} \int_{0}^{L} e^{-\int_{0}^{a} \mu(s) d s} d a=1,
$$

onde $L$ representa a idade máxima que um indivíduo pode atingir.

Não estamos considerando a imunidade derivada de anticorpos maternos e nem a transmissão vertical (sendo assim, todos os recém-nascidos são considerados suscetíveis), assim como não consideramos a perda de imunidade e a mortalidade induzida pela doença.

As considerações acima resultam no seguinte sistema de equações diferenciais parciais 


$$
\left\{\begin{array}{l}
\frac{\partial X}{\partial a}(a, t)+\frac{\partial X}{\partial t}(a, t)=-[\lambda(a, t)+\nu(a)+\mu] X(a, t) \\
\frac{\partial H}{\partial a}(a, t)+\frac{\partial H}{\partial t}(a, t)=\lambda(a, t) X(a, t)-(\mu+\sigma) H(a, t) \\
\frac{\partial Y}{\partial a}(a, t)+\frac{\partial Y}{\partial t}(a, t)=\sigma H(a, t)-(\mu+\gamma) Y(a, t) \\
\frac{\partial Z}{\partial a}(a, t)+\frac{\partial Z}{\partial t}(a, t)=\nu(a) X(a, t)+\gamma Y(a, t)-\mu Z(a, t),
\end{array}\right.
$$

com a força de infecção na idade $a$ no instante $t$ definida por

$$
\lambda(a, t)=\int_{0}^{L} \beta\left(a, a^{\prime}\right) Y\left(a^{\prime}, t\right) d a^{\prime},
$$

onde $\beta\left(a, a^{\prime}\right)$ é a taxa de contato idade-estruturada entre indivíduos suscetíveis na idade $a$ com indivíduos infecciosos da idade $a^{\prime}, \nu(a)$ é a taxa de vacinação, $\sigma^{-1}$ é o período médio de incubação e $\gamma^{-1}$ é o período médio de recuperação (ou infecção).

A partir das simplificações do modelo temos as condições de fronteira do sistema (2.2) dadas por

$$
\left\{\begin{array}{rlr}
X(0, t) & =X_{b} \\
H(0, t)=Y(0, t)=Z(0, t) & =0 .
\end{array}\right.
$$

\section{A caracterização de $\mathbf{R}_{0}$}

Resolvendo o sistema (2.2) e as condições de fronteira (2.4) no estado estacionário, e substituindo na expressão para a força de infecção dada pela equação (2.3) temos

$$
\lambda(a)=\int_{0}^{L} B^{\prime}(a, \zeta) \lambda(\zeta) e^{-\int_{0}^{\zeta} \lambda(s) d s} d \zeta
$$

onde o núcleo é dado por

$$
B^{\prime}(a, \zeta)=\sigma X_{b} e^{-\int_{0}^{\zeta} \nu(s) d s} \int_{\zeta}^{L} e^{-\sigma(s-\zeta)} e^{\gamma s}\left[\int_{s}^{L} \beta\left(a, a^{\prime}\right) e^{-(\mu+\gamma) a^{\prime}} d a^{\prime}\right] d s .
$$

As definições e os resultados matemáticos necessários para um bom entendimento do que será desenvolvido podem ser encontrados em Griffel [4] e Kreyszig [11] (análise funcional), Deimling [3] (análise funcional não-linear) e Krasnosel'skii [9] [10] (operadores integrais). Nossa intenção é apresentar os resultados estritamente necessários à compreensão do texto.

Consideremos o operador integral $T$ agindo no espaço de Banach $C[0, L]$, o conjunto de funções contínuas do intervalo $[0, L]$ em $\mathbf{R}$ com a norma usual $\|f\|=$ $\sup _{a \in[0, L]}|f(a)|$, com cone $C[0, L]^{+}=\{f \in C[0, L]: f(a) \geq 0\}$, definido pela equação $a \in[0, L]$

$$
T \lambda(a)=\int_{0}^{L} B(a, \zeta) M(\zeta, \lambda(\zeta), \nu(\zeta)) \lambda(\zeta) d \zeta
$$


onde

$$
M(\zeta, \lambda(\zeta), \nu(\zeta))=e^{-\int_{0}^{\zeta} \lambda(s) d s} e^{-\int_{0}^{\zeta} \nu(s) d s}
$$

$$
B(a, \zeta)=\sigma X_{b} \int_{\zeta}^{L} e^{-\sigma(s-\zeta)} e^{\gamma s}\left[\int_{s}^{L} \beta\left(a, a^{\prime}\right) e^{-(\mu+\gamma) a^{\prime}} d a^{\prime}\right] d s
$$

Vamos assumir que:

(i) $\beta\left(a, a^{\prime}\right)$ é contínua e positiva, exceto possivelmente em $a=a^{\prime}=0$, onde $\beta\left(a, a^{\prime}\right)$ pode ser igual a 0 , e

(ii) $\nu(a)$ é contínua ou contínua por partes com no máximo um número finito de descontinuidades, e é limitada. Então as seguintes propriedades de $M(\zeta, \lambda(\zeta), \nu(\zeta))$ e $B(a, \zeta)$ podem ser facilmente verificadas:

(a) $B(a, \zeta)$ está definida em $[0, L] \times[0, L]$ e é positiva e contínua em $a$ e $\zeta$,

(b) $M(\zeta, \lambda(\zeta), \nu(\zeta))$ está definida em $[0, L] \times[0, \infty) \times[0, \infty)$, é positiva, contínua em $\zeta$ para cada $\lambda$ e $\nu$, estritamente monótona decrescente em $\lambda$ para cada $\zeta$ e $\nu$, e existe $k_{1} \geq 0$ tal que

$$
\begin{aligned}
\mid M\left(\zeta, \lambda_{1}(\zeta), \nu(\zeta)\right) & -M\left(\zeta, \lambda_{2}(\zeta), \nu(\zeta)\right) \mid \leq k_{1}\left\|\lambda_{1}-\lambda_{2}\right\|+R\left(\lambda_{1}, \lambda_{2}\right), \\
\operatorname{com} \lim _{\left\|\lambda_{1}-\lambda_{2}\right\| \rightarrow 0} R\left(\lambda_{1}, \lambda_{2}\right) & =0, \mathrm{e}
\end{aligned}
$$
e $\nu$.

(c) existe um número real $m>0$ tal que $|M(\zeta, \lambda(\zeta), \nu(\zeta))| \leq m$ para todo $\zeta, \lambda$

Lema 1 O operador $T$ definido pela equação (3.3) é positivo, completamente contínuo e tem derivada forte de Fréchet em $0 \in C[0, L]$ na direção do cone $C[0, L]^{+}$ dada pela equação

$$
T^{\prime}(0) h(a)=\int_{0}^{L} B(a, \zeta) M(\zeta, 0, \nu(\zeta)) h(\zeta) d \zeta
$$

Além disso, $T^{\prime}(0)$ é um operador linear, completamente contínuo e fortemente positivo.

Demonstração. Na demonstração do lema usamos, além das definições, os seguintes resultados clássicos: o Critério de Compacidade (Kreyszig [11], página 407) e o Teorema de Ascoli (Kreyszig [11], página 454).

Para demonstrarmos que $R_{0}=r\left(T^{\prime}(0)\right)$ utilizamos os teoremas enunciados abaixo. Nos teoremas, $X, K$ e $T$ serão, respectivamente, espaço normado, cone e operador gerais. 
Teorema 1 (Krasnosel'skii [9], p. 135) Seja T um operador positivo (com $T(0)=$ $0)$ tendo a derivada forte de Fréchet $T^{\prime}(0)$ e a derivada assintótica forte $T^{\prime}(\infty)$, ambas com respeito ao cone. Suponhamos que o espectro do operador $T^{\prime}(\infty)$ esteja contido no círculo $|\mu| \leq \rho<1, T^{\prime}(0)$ tenha em $K$ um autovetor $h_{0}$ cujo autovalor é maior que 1 , ou seja,

$$
T^{\prime}(0) h_{0}=\mu_{0} h_{0}
$$

onde $\mu_{0}>1$ e $T^{\prime}(0)$ não tenha em $K$ autovetores correspondendo ao autovalor 1 . Se T é um operador completamente contínuo então $T$ tem pelo menos um ponto fixo não-trivial no cone.

Teorema 2 (Deimling [3], p. 228) Sejam $X$ um espaço de Banach, $K \subset X$ um cone sólido, isto é, int $(K) \neq \emptyset$, e $T: X \rightarrow X$ um operador linear, compacto $e$ fortemente positivo. Então:

(i) $r(T)>0$, onde $r(T)$ é um autovalor simples com autovetor $v \in$ int $(K)$ e não existe outro autovalor com autovetor positivo.

(ii) Se $\lambda$ é um autovalor e $\lambda \neq r(T)$ então $|\lambda|<r(T)$.

(iii) $S e S: X \rightarrow X$ é um operador linear limitado e $S x \geq T x$ em $K$, então $r(S) \geq r(T)$. Além disso, se $x \in K, x>0$ e $S x>T x$, segue $r(S)>r(T)$.

Teorema 3 (Teorema de Existência) Seja $T: C[0, L] \rightarrow C[0, L]$ o operador definido pela equação (3.3), ou seja,

$$
T u(a)=\int_{0}^{L} B(a, \zeta) M(\zeta, u(\zeta), \nu(\zeta)) u(\zeta) d \zeta .
$$

Se $r\left(T^{\prime}(0)\right) \leq 1$ então a única solução da equação

$$
\lambda(a)=\int_{0}^{L} B(a, \zeta) M(\zeta, \lambda(\zeta), \nu(\zeta)) \lambda(\zeta) d \zeta
$$

é a solução trivial. Caso contrário, se $r\left(T^{\prime}(0)\right)>1$, então existe pelo menos uma solução positiva não-trivial para esta equação.

Demonstração. Seguimos inicialmente o mesmo argumento usado por Greenhalgh [5].

Suponhamos $r\left(T^{\prime}(0)\right) \leq 1$ e que a equação (3.7) tenha uma solução positiva nãotrivial $\lambda^{*}$, isto é,

$$
\lambda^{*}(a)=\int_{0}^{L} B(a, \zeta) M\left(\zeta, \lambda^{*}(\zeta), \nu(\zeta)\right) \lambda^{*}(\zeta) d \zeta
$$

Sendo $\lambda^{*}>0$ e $M(\zeta, \lambda, \nu)$ estritamente monótona decrescente em $\lambda$, temos

$$
\int_{0}^{L} B(a, \zeta) M\left(\zeta, \lambda^{*}(\zeta), \nu(\zeta)\right) \lambda^{*}(\zeta) d \zeta<T^{\prime}(0) \lambda^{*}(a) .
$$


Como ambos os lados da equação acima são contínuos num compacto, existe $\varepsilon>0$ tal que

$$
\lambda^{*}(1+\varepsilon)<T^{\prime}(0) \lambda^{*} .
$$

Iterando a equação anterior $n$ vezes temos

$$
\lambda^{*}(1+\varepsilon)^{n}<T^{\prime}(0)^{n} \lambda^{*} .
$$

Assim

$$
\left\|\lambda^{*}(1+\varepsilon)^{n}\right\|<\left\|T^{\prime}(0)^{n} \lambda^{*}\right\| \leq\left\|T^{\prime}(0)^{n}\right\|\left\|\lambda^{*}\right\|
$$

$$
(1+\varepsilon)^{n}<\left\|T^{\prime}(0)^{n}\right\|
$$

para todo $n=1,2,3, \ldots$. Pela Fórmula de Gelfand,

$$
r\left(T^{\prime}(0)\right)=\lim _{n \rightarrow \infty}\left\|T^{\prime}(0)^{n}\right\|^{\frac{1}{n}},
$$

segue que $r\left(T^{\prime}(0)\right)>1$, o que é uma contradição.

Suponhamos agora que $r\left(T^{\prime}(0)\right)>1$. Primeiro calculamos $T^{\prime}(\infty)$. Para todo $u \in$ $K$, temos

$$
\lim _{t \rightarrow \infty} \frac{T(t u)}{t}=0
$$

desde que

$$
T(t u)=\int_{0}^{L} B^{\prime}(a, \zeta) e^{-t \int_{0}^{\zeta} u(s) d s} t u(\zeta) d \zeta,
$$

onde $B^{\prime}(a, \zeta)$ é dado pela equação (3.2), então $T^{\prime}(\infty)=0$. Agora, mostramos que $T$ é fortemente assintoticamente linear com respeito ao cone $C[0, L]^{+}$, ou seja,

$$
\lim _{R \rightarrow \infty} \sup _{\|x\| \geq R, x \in K} \frac{\left\|T x-T^{\prime}(\infty) x\right\|}{\|x\|}=0 .
$$

Note que

$$
\begin{aligned}
\|T x\| & =\sup _{a \in[0, L]}\left|\int_{0}^{L} B^{\prime}(a, \zeta) e^{-\int_{0}^{\zeta} x(s) d s} x(\zeta) d \zeta\right| \\
& \leq m^{\prime}\left[1-e^{-\int_{0}^{L} x(s) d s}\right]
\end{aligned}
$$

onde $m^{\prime}=\sup _{a, \zeta \in[0, L]}\left|B^{\prime}(a, \zeta)\right|$. Então

$$
\begin{aligned}
\lim _{R \rightarrow \infty} \sup _{\|x\| \geq R, x \in K} \frac{\left\|T x-T^{\prime}(\infty) x\right\|}{\|x\|} & =\lim _{R \rightarrow \infty} \sup _{\|x\| \geq R, x \in K} \frac{\|T x\|}{\|x\|} \\
& \leq \lim _{R \rightarrow \infty} \sup _{\|x\| \geq R, x \in K} \frac{m^{\prime}\left[1-e^{-\int_{0}^{L} x(s) d s}\right]}{\|x\|}=0,
\end{aligned}
$$


ou seja, $T$ é fortemente assintoticamente linear com respeito ao cone $C[0, L]^{+}$e sua derivada assintótica forte com respeito ao cone $C[0, L]^{+}$é $T^{\prime}(\infty)=0$.

Consideremos a identidade $\mu_{0}=r\left(T^{\prime}(0)\right)$ no Teorema 1 . De acordo com o Teorema $2, r\left(T^{\prime}(0)\right)$ é um autovalor simples de $T^{\prime}(0)$ com autovetor no int $(K)$ e não existe outro autovalor de $T^{\prime}(0)$ com autovetor positivo. Pelo argumento anterior, sendo $T^{\prime}(0)$ um operador positivo, 1 não pode ser um autovalor positivo de $T^{\prime}(0)$. Desde que $T$ é completamente contínuo, todas as condições do Teorema 1 são satisfeitas, o que resulta que a equação (3.7) tem pelo menos uma solução positiva não-trivial.

\section{A unicidade da solução não-trivial}

Nesta seção estudamos condições suficientes para que a solução não-trivial da equação (3.1) seja única e possa ser atingida por aproximações sucessivas. São utilizados resultados acerca de operadores côncavos.

Teorema 4 (Krasnosel'skii [9], p. 188) Se o operador A é u-monótono, então a equação

$$
A x=\eta x,
$$

não tem duas soluções não-nulas distintas no cone para algum valor do parâmetro $\eta$.

Teorema 5 (Krasnosel'skii [9], p. 192) Seja a equação

$$
A x=x,
$$

onde A é um operador côncavo monótono tendo uma única solução não-nula $x^{*}$ no cone K. Se uma da seguintes condições é satisfeita:

(a) $O$ cone $K$ é regular e o operador A é contínuo.

(b) O cone $K$ é normal e o operador A é completamente contínuo.

Então as sucessivas aproximações

$$
x_{n}=A x_{n-1}(n=1,2, \ldots)
$$

convergem com respeito a norma para $x^{*}$ independente da aproximação inicial $x_{0} \in$ $K, x_{0} \neq 0$.

Seja $A$ o operador agindo no espaço de Banach $C[0, L]$ com cone $C[0, L]^{+}$, definido por

$$
A u=\int_{0}^{L} B^{\prime}(a, \zeta) e^{-\int_{0}^{\zeta} u(s) d s} u(\zeta) d \zeta,
$$

com

$$
B^{\prime}(a, \zeta)=\sigma X_{b} e^{-\int_{0}^{\zeta} \nu(s) d s} \int_{\zeta}^{L} e^{-\sigma(s-\zeta)} e^{\gamma s} G(a, s) d s
$$




$$
G(a, s)=\int_{s}^{L} \beta\left(a, a^{\prime}\right) e^{-(\mu+\gamma) a^{\prime}} d a^{\prime} .
$$

A equação (4.1) pode ser reescrita como

$$
A u(a)=B^{\prime}(a, 0)+\int_{0}^{L} e^{-\int_{0}^{\zeta} u(s) d s} \frac{\partial B^{\prime}}{\partial \zeta}(a, \zeta) d \zeta
$$

de maneira que temos

$$
A u-A v=\int_{0}^{L} e^{-\int_{0}^{\zeta} v(s) d s}\left[e^{-\int_{0}^{\zeta}(u(s)-v(s)) d s}-1\right] \frac{\partial B^{\prime}}{\partial \zeta}(a, \zeta) d \zeta .
$$

Para $u>v$, se $\frac{\partial B^{\prime}}{\partial \zeta}(a, \zeta)<0$ temos $A u>A v$ e portanto o operador $A$ é monótono.

Calculando $\frac{\partial B^{\prime}}{\partial \zeta}(a, \zeta)$ temos que esta derivada parcial é dada por

$$
\begin{aligned}
\frac{\partial B^{\prime}}{\partial \zeta}(a, \zeta)= & -\sigma X_{b} e^{-\int_{0}^{\zeta} \nu(s) d s}\left\{\frac{d}{d \zeta}\left(\int_{0}^{\zeta} \nu(s) d s\right) e^{\sigma \zeta} \int_{\zeta}^{L} e^{-s(\sigma-\gamma)} G(a, s) d s\right. \\
& \left.+e^{\gamma \zeta} G(a, \zeta)-\sigma e^{\sigma \zeta} \int_{\zeta}^{L} e^{-s(\sigma-\gamma)} G(a, s) d s\right\}
\end{aligned}
$$

Observe que $\frac{\partial B^{\prime}}{\partial \zeta}(a, \zeta)<0$ se

$$
e^{\gamma \zeta} G(a, \zeta)-\sigma \int_{\zeta}^{L} e^{-\sigma(s-\zeta)} e^{\gamma s} G(a, s) d s>0 .
$$

Contudo

$$
e^{\gamma \zeta} G(a, \zeta)=\sigma \int_{\zeta}^{L} e^{-\sigma(s-\zeta)} e^{\gamma \zeta} G(a, \zeta) d s+e^{\gamma \zeta-\sigma(L-\zeta)} G(a, \zeta),
$$

portanto

$$
\begin{aligned}
& e^{\gamma \zeta} G(a, \zeta)-\sigma \int_{\zeta}^{L} e^{-\sigma(s-\zeta)} e^{\gamma s} G(a, s) d s= \\
& =e^{\gamma \zeta-\sigma(L-\zeta)} G(a, \zeta)+\sigma \int_{\zeta}^{L} e^{-\sigma(s-\zeta)}\left[e^{\gamma \zeta} G(a, \zeta)-e^{\gamma s} G(a, s)\right] d s .
\end{aligned}
$$

Sendo assim, se $e^{\gamma s} G(a, s)$ é decrescente em $s$ para todo $a$, temos $\frac{\partial B^{\prime}}{\partial \zeta}(a, \zeta)<0$. 
Teorema 6 Se a função $H(a, s)=e^{\gamma s} G(a, s)$ é decrescente em s para cada a, então o operador $A$ definido pela equação (4.1) é $u_{0}$-monótono, onde $u_{0} \equiv 1$, e completamente contínuo.

Demonstração. Que $A$ é positivo e completamente contínuo é de fácil verificação. Utilizando as observações feitas anteriormente acerca do operador $A$ e seu núcleo, a definição de $\mathrm{u}_{0}$-monótono, e o Primeiro Teorema do Valor Médio (Bartle [2], página 301 ), é possível verificar a afirmação que $A$ é u $u_{0}$-monótono, onde $u_{0} \equiv 1$.

Teorema 7 Seja a função $H(a, s)$ definida por

$$
H(a, s)=e^{\gamma s} G(a, s)
$$

decrescente em s para cada a, e o operador $T: C[0, L] \rightarrow C[0, L]$ definido pela equação (3.3). Se $r\left(T^{\prime}(0)\right)>1$ então a equação (3.1), ou seja,

$$
\lambda(a)=\int_{0}^{L} B^{\prime}(a, \zeta) e^{-\int_{0}^{\zeta} \lambda(s) d s} \lambda(\zeta) d \zeta
$$

tem uma única solução não-trivial que é atingida por aproximações sucessivas dadas por

$$
\lambda_{n}=T \lambda_{n-1},
$$

onde $n=1,2, \ldots$, e independe da aproximação inicial $\lambda_{0} \in C[0, L]^{+}, \lambda_{0} \neq 0$.

Demonstração. O operador $T$ é completamente contínuo e $\mathrm{u}_{0}$-monótono e $C[0, L]^{+}$ é um cone normal. Usando o Teorema 3 (Teorema de Existência) vemos que a equação tem pelo menos uma solução positiva não-trivial. Segue então dos Teorema 4 e Teorema 5 a convergência da seqüência (4.5) para a solução.

\section{Limites inferior e superior para $\mathbf{R}_{0}$}

O principal resultado desta seção trata da possibilidade de estimarmos limites para o raio espectral do operador $T^{\prime}(0)$, que caracteriza o número de reprodutibilidade.

Teorema 8 (Krasnosel'skii [9], p. 67) Seja um operador A linear, positivo e completamente contínuo. Seja a relação

$$
A^{p} u_{0} \geq \alpha u_{0}
$$

com $\alpha>0$, satisfeita por algum elemento não-nulo $u_{0}$ tal que

$$
-u_{0} \notin K
$$

$e$

$$
u_{0}=v-w
$$


onde $v, w \in K$ e p é algum número natural.

Então o operador $A$ tem pelo menos um autovetor $u^{*} \in K$,

$$
A u^{*}=\lambda u^{*}
$$

onde o autovalor positivo $\lambda$ satisfaz a inequação

$$
\lambda \geq(\alpha)^{\frac{1}{p}}
$$

Teorema 9 (Zabreyko [13]) Seja A um operador linear, positivo e completamente contínuo satisfazendo a inequação

$$
A^{q} v_{0} \leq \beta v_{0}
$$

onde $v_{0}$ é um elemento quase-interior do cone $K$. Então

$$
r(A) \leq(\beta)^{\frac{1}{q}},
$$

onde $r(A)$ é o raio espectral de $A$.

Teorema 10 Seja o operador linear $T^{\prime}(0)$ sobre o espaço de Banach $C[0, L]$ com cone $C[0, L]^{+}$dado pela equação (3.6), isto é,

$$
T^{\prime}(0) h(a)=\int_{0}^{L} B^{\prime}(a, \zeta) h(\zeta) d \zeta
$$

onde $B^{\prime}(a, \zeta)$ é dado pela equação (3.2).

Então

$$
\inf _{a \in[0, L]} \int_{0}^{L}\left|B^{\prime}(a, \zeta)\right| d \zeta \leq r\left(T^{\prime}(0)\right) \leq \sup _{a \in[0, L]} \int_{0}^{L}\left|B^{\prime}(a, \zeta)\right| d \zeta .
$$

Demonstração. Tomando no Teorema $8, A=T^{\prime}(0), p=1$ e $u_{0}=1$ e no Teorema $9 A=T^{\prime}(0), q=1$ e $v_{0}=1$, segue a desigualdade (5.1).

\section{Aplicando para uma taxa de contato constante}

Considerando uma taxa de contato sendo constante em todas as idades, dada por

$$
\beta\left(a, a^{\prime}\right)=\beta
$$

e a taxa de vacinação dada por

$$
\nu(a)=\nu \theta\left(a-a_{1}\right) \theta\left(a_{2}-a\right),
$$

onde $\theta$ é a função de Heaviside, $\nu$ é uma taxa de vacinação constante e $a_{1}$ e $a_{2}$ são idades tais que $0 \leq a_{1}<a_{2} \leq L$, temos que o operador 


$$
T \lambda(a)=\int_{0}^{L} \bar{B}(\zeta) e^{-\int_{0}^{\zeta} \lambda(r) d r} \lambda(\zeta) d \zeta
$$

onde

$$
\bar{B}(\zeta)=\beta \sigma X_{b} e^{-\int_{0}^{\zeta} \nu(r) d r} e^{\sigma \zeta} \int_{\zeta}^{L} e^{(\gamma-\sigma) s}\left[\int_{s}^{L} e^{-(\mu+\gamma) a^{\prime}} d a^{\prime}\right] d s,
$$

é positivo e completamente contínuo, com derivada de Fréchet no ponto 0 na direção de $C[0, L]^{+}$dada por

$$
T^{\prime}(0) \lambda(a)=\int_{0}^{L} \bar{B}(\zeta) \lambda(\zeta) d \zeta
$$

a qual é um operador fortemente positivo e completamente contínuo.

Usando o Teorema 3 (Teorema de Existência) temos o número de reprodutibilidade $R_{\nu}$ dado pelo raio espectral de $T^{\prime}(0)$, ou seja,

$$
R_{\nu}=\sup \left\{|\eta|: \eta \text { é um autovalor de } T^{\prime}(0)\right\},
$$

já que $T^{\prime}(0)$ é completamente contínuo. Pelo Teorema 2 temos $r=r\left(T^{\prime}(0)\right)$ como um autovalor simples com autovetor pertencente ao interior de $C[0, L]^{+}$(o conjunto $\left.\left\{f \in C[0, L]^{+}: f(a)>0, a \in[0, L]\right\}\right)$ e não existe outro autovalor com autovetor positivo. Considerando $h(a)=\int_{0}^{L} \bar{B}(\zeta) d \zeta$, temos

$$
T^{\prime}(0) h(a)=\int_{0}^{L} \bar{B}(\zeta)\left[\int_{0}^{L} \bar{B}(\widetilde{\zeta}) d \widetilde{\zeta}\right] d \zeta
$$

ou seja,

$$
T^{\prime}(0) h(a)=\int_{0}^{L} \bar{B}(\zeta) d \zeta \int_{0}^{L} \bar{B}(\widetilde{\zeta}) d \widetilde{\zeta}
$$

Assim

$$
T^{\prime}(0) h(a)=\eta h(a)
$$

onde

$$
\eta=\int_{0}^{L} \bar{B}(\zeta) d \zeta
$$

Então

$$
R_{\nu}=\int_{0}^{L} \bar{B}(\zeta) d \zeta
$$


isto é,

$$
R_{\nu}=\beta \sigma X_{b} \int_{0}^{L} e^{-\int_{0}^{\zeta} \nu(r) d r} e^{\sigma \zeta}\left\{\int_{\zeta}^{L} e^{(\gamma-\sigma) s}\left[\int_{s}^{L} e^{-(\mu+\gamma) a^{\prime}} d a^{\prime}\right] d s\right\} d \zeta
$$

Do Teorema 10, os limites inferior e superior de raio espectral são dados pela equação (5.1). Observemos que

$$
\int_{0}^{L} \bar{B}(\zeta) d \zeta \leq r\left(T^{\prime}(0)\right) \leq \int_{0}^{L} \bar{B}(\zeta) d \zeta
$$

ou em outras palavras, $R_{\nu}=\int_{0}^{L} \bar{B}(\zeta) d \zeta$.

Usando da função de Heaviside, a equação acima pode ser reescrita como

$R_{v}=\beta \sigma X_{b} \int_{0}^{L} e^{-\int_{0}^{\zeta} \nu(r) d r} e^{\sigma \zeta}\left\{\int_{0}^{L} e^{(\gamma-\sigma) s} \theta(s-\zeta)\left[\int_{0}^{L} e^{-(\mu+\gamma) a^{\prime}} \theta\left(a^{\prime}-s\right) d a^{\prime}\right] d s\right\} d \zeta$,

e, mudando a ordem de integração, temos

$R_{v}=\beta \sigma X_{b} \int_{0}^{L} e^{-(\mu+\gamma) a^{\prime}}\left\{\int_{0}^{L} e^{(\gamma-\sigma) s} \theta\left(a^{\prime}-s\right)\left[\int_{0}^{L} e^{-\int_{0}^{\zeta} \nu(r) d r} e^{\sigma \zeta} \theta(s-\zeta) d \zeta\right] d s\right\} d a^{\prime}$.

Resolvendo esta integral e tomando $L \rightarrow \infty$, segue

$$
R_{\nu}=\frac{\beta \sigma X_{b}}{\mu(\mu+\gamma)(\mu+\sigma)}\left\{1-\frac{\nu}{(\mu+\nu)} e^{-\mu a_{1}}\left[1-e^{-(\mu+\nu)\left(a_{2}-a_{1}\right)}\right]\right\} .
$$

Se $\nu=0$ temos

$$
R_{0}=\frac{\beta \sigma X_{b}}{\mu(\mu+\gamma)(\mu+\sigma)},
$$

que é o número de reprodutibilidade basal. Para $\nu \neq 0$, tomando $a_{1}=0$ e $a_{2} \rightarrow \infty$, temos

$$
R_{\nu}=\frac{\beta \sigma X_{b}}{(\mu+\nu)(\mu+\gamma)(\mu+\sigma)} .
$$

Além disso, sendo

$$
\begin{aligned}
G(a, s) & =\int_{s}^{L} \beta\left(a, a^{\prime}\right) e^{-(\mu+\gamma) a^{\prime}} d a^{\prime} \\
& =\int_{s}^{L} \beta e^{-(\mu+\gamma) a^{\prime}} d a^{\prime}=\frac{\beta e^{-(\mu+\gamma) s}}{(\mu+\gamma)}-\frac{\beta e^{-(\mu+\gamma) L}}{(\mu+\gamma)},
\end{aligned}
$$


temos

$$
\begin{aligned}
e^{\gamma s} G(a, s) & =e^{\gamma s}\left(\frac{\beta e^{-(\mu+\gamma) s}}{(\mu+\gamma)}-\frac{\beta e^{-(\mu+\gamma) L}}{(\mu+\gamma)}\right) \\
& =\frac{\beta}{(\mu+\gamma)}\left[e^{-\mu s-\gamma s+\gamma s}-e^{-\mu L-\gamma L+\gamma s}\right] \\
& =\frac{\beta e^{-\mu s}}{(\mu+\gamma)}\left[1-e^{-(\gamma+\mu)(L-s)}\right],
\end{aligned}
$$

que é uma função decrescente em $s$ para todo $a \in[0, L]$. Assim, quando $R_{\nu}>1$ existe uma única solução não-trivial para a força de infecção que pode ser atingida pela seqüência $\lambda_{n}=T \lambda_{n-1}$, onde $\lambda_{0} \in C[0, L]^{+}$e $\lambda_{0} \neq 0$ é qualquer aproximação inicial.

Para obtermos o esforço mínimo de vacinação a fim de erradicar a doença é necessário calcular o infimum do conjunto $\left\{\nu: R_{\nu}<1\right\}$,ou seja,

$$
\nu^{t h}=\inf \left\{\nu: R_{\nu}<1\right\} \text {. }
$$

Esta condição pode ser aplicada à equação (6.2), tomando $R_{\nu}=1$, e resolvendo esta equação em $\nu$. Desta forma obtemos $\nu^{\text {th }}$ sempre que este valor existir.

\section{Conclusão}

O principal objetivo deste trabalho é a busca de condições para que a equação integral para $\lambda(a)$, dada pela equação (3.1), com o núcleo $B^{\prime}(a, \zeta)$, dado pela equação (3.2), tenha uma solução não-trivial. Em nosso trabalho, utilizando uma taxa de contato idade-estruturada $\beta\left(a, a^{\prime}\right) \in C[0, L]^{+}$, onde $\beta(0,0)$ pode ser igual a zero, mostramos que se o raio espectral do operador $T^{\prime}(0)$ é maior que 1 , então a equação (3.1) tem pelo menos uma solução não-trivial. Caso contrário, ou seja, se $r\left(T^{\prime}(0)\right)$ é menor ou igual a 1 , a única solução da equação (3.1) é a solução trivial.

A questão da unicidade da solução não-trivial de (3.1) é respondida pelo Teorema 7. Quando $r\left(T^{\prime}(0)\right)>1$ e a função $H(a, s)$ é decrescente em $s$ para cada $a$, temos que a solução não-trivial da equação integral (3.1) é única e pode ser atingida recursivamente pela seqüência $\lambda_{n}=T \lambda_{n-1}, n=1,2, \ldots$, onde $\lambda_{0} \in C[0, L]^{+}$, e $\lambda_{0} \neq 0$ é qualquer aproximação inicial.

Dado que, geralmente, a obtenção do raio espectral é uma tarefa difícil, a possibilidade de estimativas para o mesmo é conveniente. O Teorema 10 fornece limitantes superior e inferior para $r\left(T^{\prime}(0)\right)$ que, em particular para o caso de taxa de contato constante, são iguais ao valor exato de $r\left(T^{\prime}(0)\right)$.

É interessante notar que, biologicamente, a equação integral (3.1) ter solução não-trivial equivale a que uma dada doença seja capaz de invadir e se estabelecer em uma certa comunidade, uma vez que $\lambda(a)$ representa a força de infecção, ou seja, a taxa per capita de novos casos por unidade de tempo. Temos então caracterizado o Número de Reprodutibilidade Basal como o raio espectral do operador $T^{\prime}(0)$, uma vez que se o mesmo é maior que 1 a doença se instala na população e se é menor 
ou igual a 1 a doença se extingüe. Em particular, considerando $\nu=0$ obtemos o Número de Reprodutibilidade Basal, o tão procurado $R_{0}$.

Uma vez que, por sua própria definição, a força de infecção é passível de alterações por meio de intervenções na população (por exemplo, quimioterapias, campanhas de vacinação, etc.), a obtenção de $\lambda$ é interessante quando a partir dela é possível obter $\beta\left(a, a^{\prime}\right)$, este sim um parâmetro que caracteriza o padrão de comportamento da doença na comunidade em questão. Quando temos não só a unicidade mas também uma maneira de obter $\lambda$, no nosso caso via uma seqüência recursiva, podemos, ao estudar o caso de uma população em equilíbrio em relação ao tempo e que não recebeu qualquer intervenção de controle relativamente a uma dada doença, calcular os vários parâmetros envolvidos na taxa de contato $\beta\left(a, a^{\prime}\right)$.

Finalmente, a grande importância da obtenção de uma expressão que caracterize o Número de Reprodutibilidade $R_{\nu}$, via raio espectral, reside no fato de podermos avaliar o esforço de vacinação necessário à erradicação da doença (uma vez que a caracterização foi obtida para uma função $\nu$ contínua ou contínua por partes com no máximo um número finito de descontinuidades, e limitada, veja condição (ii)). Particularmente, no caso de $\beta\left(a, a^{\prime}\right)$ ser constante, vemos que a expressão obtida para o Número de Reprodutibilidade permite avaliar o valor exato do esforço mínimo para a erradicação da doença. O valor do esforço mínimo da vacinação pode ser obtido da equação (6.2) fazendo $R_{\nu}=1$.

\section{Referências}

[1] R.M. Anderson and R.M. May, "Infections Diseases of Humans: Dynamics and Control", Oxford University Press, New York, 1992.

[2] R.G. Bartle, "The Elements of Real Analysis", John Wiley \& Sons, New York, 1964.

[3] K. Deimling, "Nonlinear Functional Analysis", Springer-Verlag, Berlin, 1985.

[4] D.H. Griffell, "Applied Functional Analysis", Ellis Horwood Limited, Chichester, England, 1981.

[5] D. Greenhalgh, Threshold and stability results for an epidemic model with an age-structured meeting rate, IMA J. Math. Appl. Med. Biol., 5 (1988), 81-100.

[6] D. Greenhalgh, Vaccination Campaigns for Common Childhood Diseases, Math. Biosc., 100 (1990), 201-240.

[7] R. Hoppenstead, An age dependent epidemic model, J. Franklin Inst., 297 (1974), 325-333.

[8] H. Inaba, Threshold and Stability Results for an Age-structured Epidemic Model, J. Math. Biol., 28 (1990), 411-434. 
[9] M.A. Krasnosel'skii, "Positive Solutions of Operator Equations", P. Noorddhoff ltda. Groningen, The Netherlands, 1964.

[10] M.A. Krasnosel'skii, "Topological Method in the Theory of Nonlinear Integral Equation", Pergamon Press, Oxford, 1964.

[11] E. Kreyszig, "Introductory Functional Analysis with Applications", John Wiley \& Sons, New York, 1989.

[12] H.M. Yang, Directly transmitted infections modeling considering an agestructure contact rate - epidemiological analysis, Math. Comp. Mod., 29 (1999), $11-30$.

[13] P.P. Zabreyko, M.A. Krasnosel'skii, and V. Y. Stecenko, Bounds for the Spectral Radius of Positive Operator, Math. Notes, 1, 3 and 4 (1967), 306-310. 
